\title{
Towards Heralded Two-Photon Absorption and Two-Photon Excited Fluorescence for Quantum Microscopy and Spectroscopy
}

\author{
Andreas Jechow ${ }^{1,2,3}$ \\ ${ }^{1}$ University of Potsdam, Karl-Liebknecht Str. 24-25, 14476 Potsdam, Germany \\ ${ }^{2}$ Remote Sensing, German Research Centre for Geoscience (GFZ), Telegrafenberg, \\ 14473 Potsdam, Germany $\ddagger$ \\ ${ }^{3}$ Ecohydrology, Leibniz Institute of Freshwater Ecology and Inland Fisheries (IGB), \\ Müggelseedamm 310, 12587 Berlin, Germany $\S$ \\ E-mail: andreas.jechow@gmx.de
}

\begin{abstract}
The interaction between single or a fixed number of photons with a single absorber is of fundamental interest in quantum technology. The harnessing of light matter interactions at the single particle limit has several potential applications ranging from quantum communication and quantum metrology to quantum imaging. In this letter, a setup for heralded two-photon absorption at the single absorber level is proposed. The setup is based on a heralded two-photon source utilizing spontaneous parametric down-conversion, entanglement swapping and sum frequency generation for joint detection. The feasibility of the scheme is discussed by reviewing recent achievements in utilizing entangled and correlated photons for two-photon absorption as well as single photon absorption experiments at the limit of single absorbers in the context of applications in imaging (here mainly microscopy) and spectroscopy.
\end{abstract}

Keywords: multi-photon processes; quantum optics; photon statistics; fluorescence

$\ddagger$ current affiliation

$\S$ current affiliation 


\section{Introduction}

For many decades, "nonlinear optics" was attributed to be the study of light matter interactions at high light intensities involving many photons [1]. However, with the advent of both atomic physics and quantum optics experiments involving single particles of light and matter [2, 3, 4], the field of nonlinear optics has recently also extended towards low intensities down to the single photon level $[5,6]$. The interaction between single absorbers and single (or a fixed number of) photons is of fundamental scientific interest and promises to unlock many potential applications in the future. However, it bears also several challenges. In this letter, a setup for two-photon absorption (TPA) $[7,8]$ or two-photon excited fluorescence (TPEF) utilizing a heralded two-photon source is proposed. The proposed scheme has potential applications in quantum microscopy and quantum spectroscopy.

This short communication is structured as follows: after a brief review of background information, the proposed setup is presented and the feasibility is discussed. In the appendix, more background information are given and the relevant fundamentals as well recent experimental and theoretical work are reviewed.

\section{Background}

\subsection{Two-photon microscopy at low intensities}

Two-photon microscopy (TPM) is one of the state of the art high resolution imaging technologies used in the life sciences sector [9] exploiting TPEF. The advantages of twophoton microscopy are the deeper penetration depth into the tissue and a drastically increased depth resolution (see for example review article by Zipfel et al. [10]). It enabled in vivo deep brain imaging, which is of great interest in neuroscience as it made the efficient detection of beta-Amyloid plaque, a protein linked to Alzheimers disease, possible [11]. Thus far, TPM is exclusively performed with classical light at relatively high light intensities. However, biological samples are very sensitive to optical damage [12] by high intensity light and therefore reducing the optical load at the probe is paramount to prevent irreversible damage to living cells. Usually, this is done by using ultrashort pulses in TPM setups that still contain many photons. In the context of quantum nonlinear optics the question arises: "To what extend can TPA or TPEF be observed with extremely weak fields down to the two photon limit?".

Several theoretical proposals for enhanced TPA exist and progress in experimental work has been made. Three main strategies can be formulated:

- utilize light with a tailored photon statistics,

- utilize absorbers with an enhanced absorption cross section or enhanced fluorescence,

- facilitate strong photon-absorber coupling,

with the latter two being somehow interlinked. 


\subsection{Tailored photon statistics for enhanced TPA}

The photon statistics of a light field is important for nonlinear processes as outlined comprehensively by Mollow [13]. Enhanced TPA was experimentally achieved with "classical" light by utilizing thermal light instead of coherent light $[14,15]$. The utilization of entangled photons for TPA was proposed by Gea-Banacloche [16] as well as Javaninen and Gould [17] and later experimentally demonstrated with atoms [18] as well as molecules $[19,20,21,22]$. Other nonlinear interactions utilizing entangled photons include for example sum frequency generation (SFG) [23]. Entangled or correlated photons are still most commonly generated by spontaneous parametric down-conversion (SPDC). Highly efficient SPDC sources can be realized by using periodically poled materials with waveguide structures [24]. Heralded single photon sources based on SPDC [25] as well as the generation of multi-partite photon entanglement by utilizing entanglement swapping [26] or joint detection have been demonstrated. Recent work includes the creation of photon triplets [27]. Guerreiro et al. have done seminal work on detecting of weak nonlinear interactions between single photons using a heralded scheme comprising multiple nonlinear crystals [28, 29]. Spasibko et al. could recently demonstrate enhanced multiphoton processes using a pulsed light source of bright squeezed vacuum [30].

\subsection{Tailored absorbers for enhanced TPA or TPEF}

In TPM, usually $\pi$-conjugated chromophores with strong electron-donor and acceptor groups that exhibit enhanced TPA cross sections are used. Such chromophores can be tailored by designing donor acceptor groups, $\pi$-conjugated connectors and branching symmetry. A review on TPA chromophores with dipolar, quadrupolar, octupolar and more extended branched structures can be found in [31]. Other ways to increase the fluorescence or absorption yield include the exploitation of plasmonic effects on surfaces [32] or by utilizing nanoparticles [33]. Such nanoparticles can have tailored shapes and serve as nanoantennas. Besides enhanced absorption cross section it to improve or even tailor the fluorescence by plasmonic effects [34].

\subsection{Strong atom-photon coupling}

To achieve strong photon-absorber coupling, several schemes have been implemented. Most prominently, high-Q enhancement cavities have been used to ensure that the photon interaction with an absorber is enhanced [6]. However, while such cavities are excellent for fundamental tests in quantum optics, they are not suitable for many real world applications as they require extreme stability and lack scalability. For TPM applications, cavities afre not well suited. Therefore, several optical interconnects for free space coupling of photons and single absorbers have been proposed and utilized including the use of high NA lenses [35, 36, 37, 38] or mirrors for trapped ions [39, 40, 41] or molecules [42]. 


\section{Heralded two-photon absorption setup}

Here, a new scheme for heralded TPA experiments, exploiting several of the aforementioned schemes altogether, is proposed with the emphasis on applications in spectroscopy or microscopy. The proposed setup to study TPA or TPEF utilizing a heralded two-photon source is depicted in Fig. 1. It is inspired by the setup used by Guerrero et al. that targeted applications in quantum communication [29].

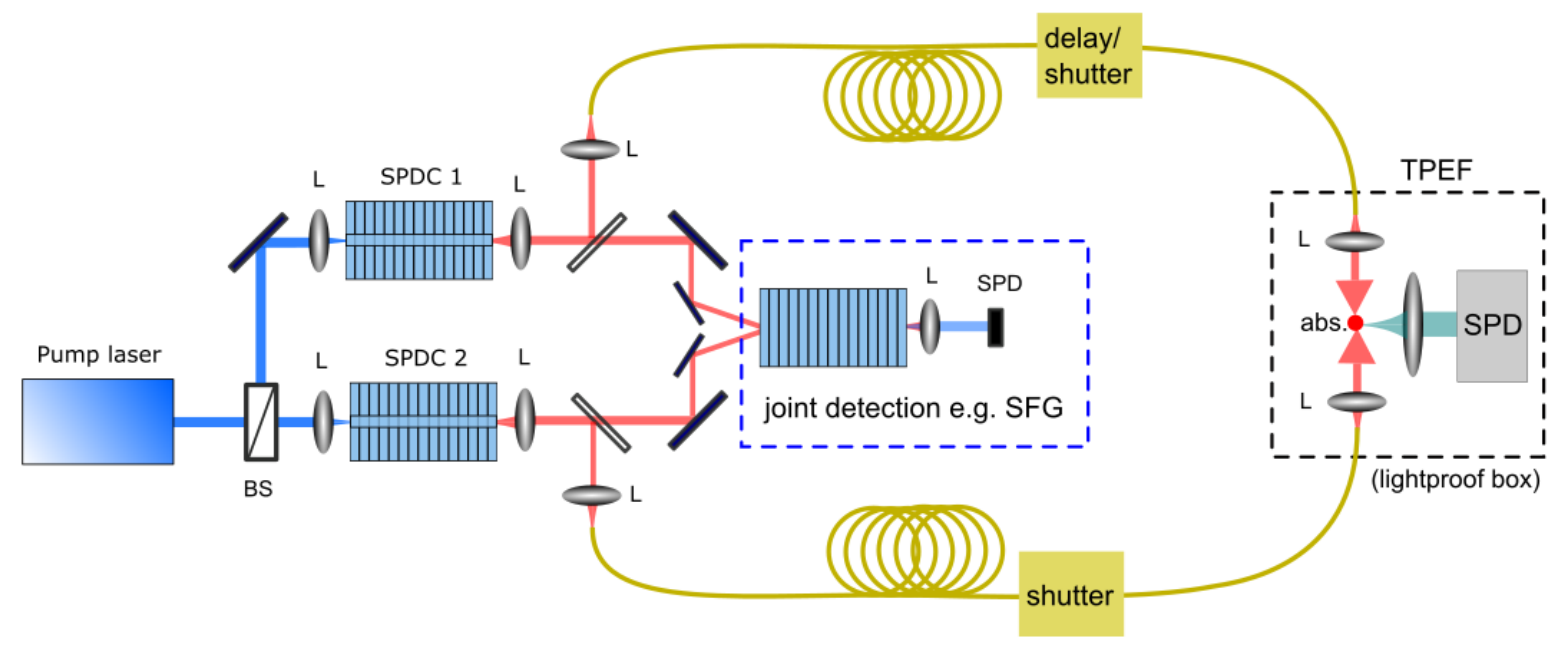

Figure 1. Sketch of a two-photon absorption (TPA) or two-photon excited fluorescence (TPEF) experiment utilizing photon pairs from a heralded two-photon source. Two waveguide SPDC sources (SPDC1 and SPDC2) are utilized and a joint detection of signal from one source and idler from the other source is performed in a waveguide nonlinear crystal by SFG and subsequent detection of an up-converted photon at a single photon detector (SPD). If two photons are jointly detected at the SFG crystal, two correlated/entangled photons must be in the two other channels. The delay between these two heralded photons can be controlled in fibers and/or by other means. The two-photons are reunified at a two-photon absorber. In the TPEF setup, the absorber will emit a fluorescence photon that can be detected with another SPD. The detection of the up-converted photon behind the SFG can herald the presence of two other photons that then can trigger two-photon excited fluorescence (TPEF) in a gated detection setup to reduce noise.

SPDC in two separate nonlinear periodically poled waveguide crystals (SPDC1 and SPDC2) is pumped by a common pump laser. The pump laser radiation is separated into two beams at a beam splitter (BS) and subsequently coupled into the waveguide crystals using high NA lenses (see e.g. [43] using periodically poled lithium niobate). In each of the waveguides a certain amount of photon pairs are generated independently, depending on the material constant, the phase matching conditions, the waveguide architecture (length, width etc.) as well as the pump power. The light exiting the waveguides is collimated and the pump fields are filtered out using bandpass filters.

In the next step, the signal and idler fields from the down-converted light fields created in each SPDC crystals are separated utilizing dichroic filters. Subsequently, the 
idler field from SPDC crystal 1 and the signal field from SPDC crystal 2 are directed towards a third periodically poled nonlinear waveguide crystal, where the two light fields are spatially overlapped and coupled into the waveguide.

Behind this waveguide crystal a single photon detector is placed behind a bandpass filter. In this crystal up-conversion of the two independently generated broadband downconverted fields occurs and a single up-converted photon is generated if two photons of the matching energy arrive at the waveguide crystal "simultaneously". Therefore, only if two photon pairs are generated "simultaneously" in the SPDC1 and SPDC2 crystals, a joint detection of idler and signal photons from different SPDC crystals is possible [29]. This heralds the presence of two entangled photons in the other idler and signal fields.

This so called "faithful" entanglement swapping scheme was proposed by Sangouard et al. in 2011 [44] and could recently be shown by Guerreiro et al. in the telecommunication wavelength regime in the framework of quantum communication [29].

In contrast to the setup of Guerreiro et al., the signal field from SPDC crystal 1 and the idler field from SPDC crystal 2 on the other hand are coupled into fiber optics with integrated optical shutters that are opened when a photon pair is heralded at the SFG crystal. Both fiber optics require an adequate length to compensate for the slow electronics that control these shutters. At least one of the two paths requires an additional tunable optical delay line to provide fine tuning ability of any optical path length difference between the two photons. Each optical field from the fiber optics is then directed towards a TPEF setup, where the two light fields are spatially overlapped and focused onto an absorber. The absorber is ideally a levitating single molecule with a large TPA cross section or an aggregate of chromophores on a plasmonic surface. One way to implement the levitating single particle is to use a Paul trap architecture as shown with fluorescent dyes [45], nanoparticles [46] and proposed for large biomolecules [47]. Fluorescence of the absorber is collected by a high NA optic [42], that could possibly be a diffractive optics [48], or a (tapered) fiber [45, 49]. On the detector side, a single photon detector or a sensitive camera [15] could be used.

\section{Discussion and conclusion}

The proposed setup could be applied to study TPA with few photons in detail. One major application is TPEF for microscopy, where the use of entangled photons (biphotons) promise a drastic signal enhancement (see e.g. [21, 22] and more details in appendix). Such enhancements have also been demonstrated recently in multiphoton processes up to the 4th order [30]. The setup could be utilized to test entangled twophoton absorbers, as an enhanced signal with classical light could stem from a cascaded single-photon process and not from a real TPA process. Then, the entanglement would not provide a benefit [50]. By harnessing the absorption of only two photons in a heralded setup and by controlling the delay between the two entangled photons it 
appears also feasible to perform virtual state spectroscopy $[51,52]$.

The setup relies on well known components and the entanglement swapping was recently demonstrated [29]. Although the individual building blocks have not been tested altogether there seems to be no obvious reason to rule out their combined functioning. However, the implementation remains very challenging. One obstacle is the detection efficiency and dark counts of the detectors. Guerreiro et al. used stock single photon avalanche diodes with relatively high dark count rate. With cryogenic detectors or single photon detectors based on superconducting nanowires [53], a lower dark count rate might be feasible.

In conclusion, the study of TPA and TPEF with only very few or even a defined number of photons utilizing the scheme proposed here appears feasible with current technical devices. Although the complexity of the setup and the expected low signal prevent an immediate real-world application in TPM, the setup provides a good tool for fundamental studies in the context of quantum microscopy and quantum spectroscopy $[52]$.

\section{Appendix A. Photon statistics and nonclassical light sources}

\section{Appendix A.1. Photon bunching}

Different light sources emit light with different photon statistics. Generally, classical light will have a rather random temporal distribution of photons (Fig A1 middle graph). On the one hand, coherent light obeys Poissonian counting statistics on the other hand thermal light obeys Bose Einstein counting statistics. The degree of second-order coherence of a light field at a time delay $\tau$ with respect to a time $t$ is a measure of the two-photon correlation and is defined as:

$$
g^{(2)}(t, \tau)=\frac{\left\langle E^{+}(t) E^{+}(t+\tau) E^{-}(t+\tau) E^{-}(t)\right\rangle}{\left\langle E^{+}(t) E^{-}(t)\right\rangle^{2}},
$$

with $E^{+}$being the positive and $E^{-}$being negative frequency parts of the field and \langle\rangle

being the quantum expectations. For a perfectly coherent single-mode light field of constant amplitude the degree of second-order coherence will always be one:

$$
g_{\text {coherent }}^{(2)}(t, \tau)=g_{\text {coherent }}^{(2)}(0)=1 \text {. }
$$

For a thermal (or chaotic) light field, the degree of second-order coherence will reach a value of two at zero time delay and will drop to lower values for larger time delays:

$$
g_{\text {thermal }}^{(2)}(t, \tau)<g_{\text {thermal }}^{(2)}(0)=2 .
$$

Thus, the probability of detecting two photons at zero time delay with thermal light is twice as high as for coherent light. More than 60 years ago and even before the laser was invented, Hanbury Brown and Twiss have famously experimentally observed this so called "photon bunching" [54]. However, at large time delays $\tau$, the degree of 
second-order coherence for the thermal and coherent light field will become equal, both reaching values of one

$$
g_{\text {coherent }}^{(2)}(t, \tau)=g_{\text {thermal }}^{(2)}(t, \tau)=1 .
$$

As typical coherence times of thermal like radiation are on the order of femtoseconds, it took more than 50 years until photon bunching on such ultrafast timescales was observed due to limited detector bandwidths. In 2009, Boitier et al. exploited two-photon absorption in a semiconductor in combination with an interferometric auto-correlation measurements to unravel photon bunching with femtosecond temporal resolution [14].

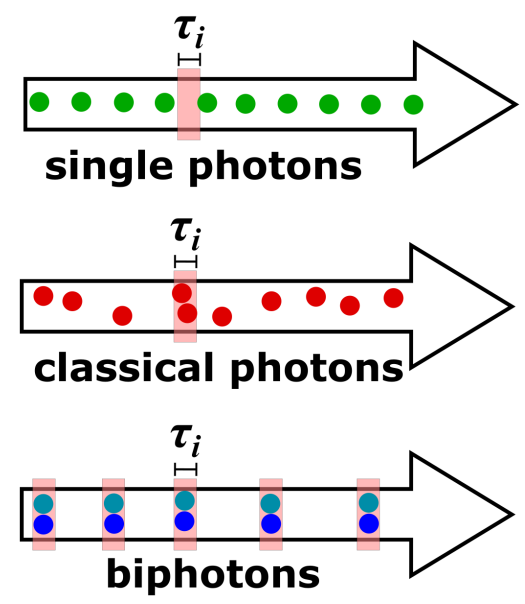

Figure A1. Illustration of the correlation of the emission from different light sources with respect to a time window $\tau_{i}$. Upper graph: perfect single photon source with no two-photon correlations, middle graph: classical light source with random two-photon correlation and lower graph: perfect two-photon source that emits photon pairs or "biphotons" with perfect two-photon correlations.

Quantum light (or "nonclassical" light) can have a tailored photon statistics. Today nonclassical light sources are widely used in quantum optics, especially for quantum cryptography, quantum computing and quantum communication.

\section{Appendix A.2. Photon pair sources}

An ideal two-photon source always emits two photons at a time (see Fig A1 lower graph) and the probability to find two photons at a time will be maximized, at least for low photon flux densities. Entangled photon pairs or "biphotons" can have such strong spatio-temporal correlations $[55,56]$ and have opened up many key findings in the field of quantum optics. They are most commonly generated by spontaneous parametric down conversion (SPDC) in a nonlinear optical crystal $[57,58,59]$. In SPDC, one pump photon with a high energy will decay into two photons of lower energy. Historically, one photon is called "idler" and the other is called "signal". Momentum and energy conservation applies so the sum of the energies of signal and idler equals that of the original pump 
photon. The peculiar behavior of these biphotons have been utilized to demonstrate stunning experiments impossible with classical light e.g. quantum teleportation [60]. Entangled photon pairs can also be obtained from semiconductors such as a quantum dot embedded in an LED [61], by four-wave-mixing [62], SPDC in semiconductors with Bragg waveguides [63] or from on chip SPDC in a laser diode with a super-lattice structure $[64,65]$. However, most of these systems are still under development and not mature, yet.

\section{Appendix A.3. Single photon sources}

An ideal single photon source (Fig A1 upper graph) always emits just one photon at a time and the chance to find two photons at a time will be zero, which is called "antibunching". Within the last few years, a lot of progress was made with such single photon sources [66]. Single photons can be obtained from heralded sources exploiting parametric down-conversion $[25,67,68]$ or single emitters like single molecules [69, 70], single atoms $[71,72]$, quantum dots $[73,74,75]$, silicon nanowires [76], silicon carbide [77] or nitrogen vacancies in diamond $[78,79]$.

\section{Appendix B. Two-photon absorption (TPA)}

\section{Appendix B.1. Classical TPA}

Two-photon absorption (TPA) is referred to be the "simultaneous" || absorption of two photons by an absorber. First a photon interacts with an absorber and brings an electron from a ground state $\left|\psi_{g}\right\rangle$ with an energy eigenvalue of $\varepsilon_{g}$ to an intermediate (nonresonant) state $\left|\psi_{i}\right\rangle$. This intermediate state has a very short lifetime $\tau_{i}$ (on the order of femtoseconds) and a complex energy eigenvalue of $\varepsilon_{i}+i k_{i} / 2$. If a second photon arrives within that lifetime window, the electron can be excited to the final state $\left|\psi_{f}\right\rangle$ with an energy eigenvalue of $\varepsilon_{f}$. In the case of two-photon excited fluorescence (TPEF) a fluorescence photon is emitted subsequently to the absorption process (see Fig. B1).

Maria Göppert-Mayer derived two-photon absorption theoretically in her thesis in 1931 using quantum theory and the Dirac notation [7]. The first experimental observation of TPA was achieved 30 years after the theoretical prediction and soon after the invention of the laser [8]. Garrett and Kaiser observed fluorescence at 425 $\mathrm{nm}$ induced by light with a longer wavelength of $694 \mathrm{~nm}$. This was one milestone in the field of nonlinear optics. Since then, multi-photon processes in general, and two-

photon processes in particular, have been studied in great depth both theoretically and experimentally. Today, multi-photon absorption processes are utilized in a wide array of applications for example laser scanning multi-photon microscopy [9].

\| It should be noted, that this is of course an unphysical term. Here, simultaneous means that the two-photons have to arrive within the lifetime of the intermediate state. 


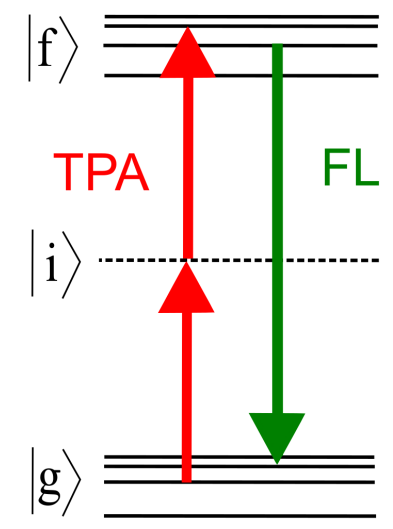

Figure B1. Sketch of two-photon excited fluorescence (TPEF). Two-photon absorption (TPA) occurs from a ground state $\left|\psi_{g}\right\rangle$ to a final state $\left|\psi_{f}\right\rangle$ via an intermediate state $\left|\psi_{i}\right\rangle$ with an intermediate state lifetime $\tau_{i}$, followed by the subsequent emission of a fluorescence photon (FL).

The TPA rate, $R_{T P A}$, will depend quadratically on the intensity $I$ or the photon flux density $\phi$ of the light field, when using a classical light field:

$$
\begin{aligned}
& R_{T P A} \propto I^{2}, \\
& R_{T P A}=\delta_{T P A} \cdot \phi^{2},
\end{aligned}
$$

with the classical TPA absorption cross section $\delta_{T P A}$ :

$$
\left.\delta_{T P A}=\frac{\pi}{2} \omega_{1} \omega_{2} \delta\left(\varepsilon_{f}-\varepsilon_{g}-\omega_{1}-\omega_{2}\right) \times\left|\sum_{j}\left[\frac{D_{21}^{(i)}}{\Delta_{1}^{(i)}-i \kappa_{i} / 2}+\frac{D_{12}^{(i)}}{\Delta_{2}^{(i)}-i \kappa_{i} / 2}\right](\mathrm{B}) \cdot\right|^{2}\right)
$$

being on the order of:

$$
\delta_{T P A} \approx \sigma_{g i} \sigma_{i f} \cdot \tau_{i}
$$

Here, $\sigma_{g i}$ and $\sigma_{i f}$ are the one-photon absorption cross sections, $\omega_{1}$ and $\omega_{2}$ are the frequencies of the photons (or fields) driving the TPA transition, $\varepsilon_{f}$ and $\varepsilon_{g}$ are the energy states of the final and the ground state, respectively, $\Delta_{k}^{(i)}=\varepsilon_{i}-\varepsilon_{g}-\omega_{k}$ is an energy mismatch factor and $D_{21}$ and $D_{12}$ are the transition matrix elements $D_{k l}^{(i)}=\left\langle\psi_{f}\left|d_{k}\right| \psi_{i}\right\rangle\left\langle\psi_{i}\left|d_{l}\right| \psi_{g}\right\rangle$ with the electric dipole components $d_{k}, d_{l}$ with $k, l=1,2$.

\section{Appendix B.2. Enhanced classical TPA}

Mainly motivated to reduce photo-damage, a lot of effort has been made on the design of new absorbers with a large two-photon cross section by using molecules with quadrupolar or more complex structures [80] including dendrimers [20]. Furthermore, it was shown that by coupling fluorescent dyes to plasmonic nanostructures, such as spheres, it is possible to achieve a field enhancement resulting in an increased absorption as well as increased fluorescence of dye molecules [81]. These nanostructures can also be fabricated 
as functional surfaces or in more complex shapes and can serve as "optical antennas" $[82,83]$. The field enhancement depends on the wavelength, the size of the nano-particle and also on the distance between the absorber and the fluorescent dye. In some cases a fluorescence quenching was observed, therefore the spacer layer of the nano-particles (as sketched in Fig. B2) has to be carefully adjusted depending on the dye and the excitation wavelength.

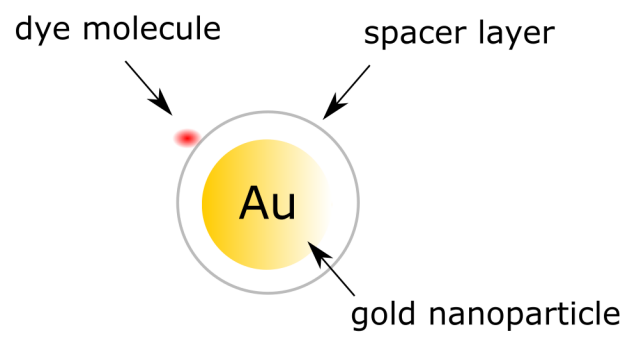

Figure B2. Sketch of a dye molecule attached to a metal nanoparticle (here gold) with a spacer layer to exploit plasmonic enhancement of absorption or fluorescence.

\section{Appendix B.3. TPA and photon statistics}

It is well known, that higher order absorption processes will probe higher order correlation functions of the light fields [84, 85, 86, 13, 87]. In 1968 Mollow used pertubative theory to connect the degree of second order coherence with the TPA rate [13]. Mollow assumed, that the spectral width of the final excited energy state is large compared to the bandwidth of the field $\Delta \omega_{f} \gg \Delta \omega$, as typical for commonly used laser dye molecules. Mollow showed, that for weak stationary light fields the TPA rate is linearly proportional to the degree of second-order coherence function at zero time delay

$$
R_{T P A}(\omega)=g^{(2)}(0)\left|D\left(\omega_{0}\right)\right|^{2} 2 \frac{\Delta \omega_{f} / 2}{\left(\Delta \omega_{f} / 2\right)^{2}+\left(2 \omega-\omega_{f}\right)^{2}} I^{2},
$$

with $I$ being the intensity of the light field, $\omega_{f}$ being the transition frequency, $\Delta \omega_{f}$ being the spectral full width at half maximum of the final state and $D(\omega)$ transition.

For this work, only the influence of the photon statistic on the TPA rate is of and interest. Thus, this can be simplified to:

$$
R_{T P A}(\omega)=g^{(2)}(0) C I^{2},
$$

with $C$ being a constant that incorporates the properties of the absorber. When comparing the TPA rates of coherent and thermal light one finds

$$
\frac{R_{T P A}^{\text {thermal }}(\omega)}{R_{T P A}^{\text {coherent }}(\omega)}=\frac{g_{\text {thermal }}^{(2)}(0)}{g_{\text {coherent }}^{(2)}(0)}=2 .
$$

In seminal work, Boitier et al. have recently demonstrated, that amplified spontaneous emission (ASE) can exhibit intensity correlations on an ultrashort timescale similar to thermal radiation [14]. They unraveled the photon bunching effect by TPA in a 
photo-multiplier tube utilizing an interferometric setup. This was the first experimental validation of Mollows and others predictions.

Jechow et al. [15] could later demonstrate that an enhanced signal for two-photon excited fluorescence (TPEF) can be achieved when utilizing the photon statistics of the excitation light field. They used ASE from a superluminescent diode [88] in comparison to coherent light [15] and measured the fluorescence from common fluorophores and quantum dots in solution. The ASE was characterized by a thermal like photon statistics and the TPEF rate was directly proportional to the measured degree of second order coherence of the light utilized light sources. This represents a principal step towards utilizing the photon statistics in multi-photon microscopy.

It has to be pointed out, that real "classical" light sources can emit light with a photon statistics being a mixture of coherent and thermal like. Several studies have been carried out with semiconductor light sources such as high power superluminescent diodes [89, 90].

The discussion here is mainly restricted to TPA. However, photon bunching is not limited to two-photon processes and the degree of second-order coherence of a light field can be extended to arbitrarily high orders $n$ [91]. Starting with the more general form of $g^{(2)}(t, \tau)$ with the photon number operator $\hat{n}=\hat{a}^{\dagger} \hat{a}$ we get:

$$
g^{(2)}(t, \tau)=\frac{\langle: \hat{n}(t) \hat{n}(t+\tau):\rangle}{\langle\hat{n}(t)\rangle\langle\hat{n}(t+\tau)\rangle} .
$$

Extending this equation to the degree of n-th order coherence yields:

$$
g^{(n)}\left(t, \ldots t_{n}\right)=\frac{\left\langle: \prod_{i=1}^{n} \hat{n}\left(t_{i}\right):\right\rangle}{\prod_{i=1}^{n}\left\langle\hat{n}\left(t_{i}\right)\right\rangle} .
$$

Thus, the probability to find $\mathrm{n}$ photons at zero time delay is $n$ ! times greater than at larger time delays

$$
1 \leq g_{\text {thermal }}^{(n)}\left(t, \ldots t_{n}\right)<g_{\text {thermal }}^{(n)}(0)=n !
$$

while for coherent light it remains always 1 for all time delays. Extending Mollows wrok, Agarwal [87] showed that the multi-photon absorption rate scales directly proportional to $g^{(n)}(0)$ resulting in an $n$ ! enhancement when using thermal light for multi-photon processes.

\section{Appendix B.4. Entangled Two-Photon Absorption}

Two-photon absorption with entangled photons was first experimentally demonstrated with atoms [18] and later with molecules [19, 20, 21, 22]. Theoretical work predicted that TPA with entangled photon pairs at low flux rates will show a linear intensity dependence (see Fig. B3), contrary to classical light [16, 17], which was proven experimentally by Dayan et al. using broadband biphotons [23].

Subsequent theoretical work by Dayan [92] generalized for two-photon processes showed that below a critical flux of $\Phi_{x} \approx \Delta_{S P D C}$, with $\Delta_{S P D C}$ being the bandwidth of 


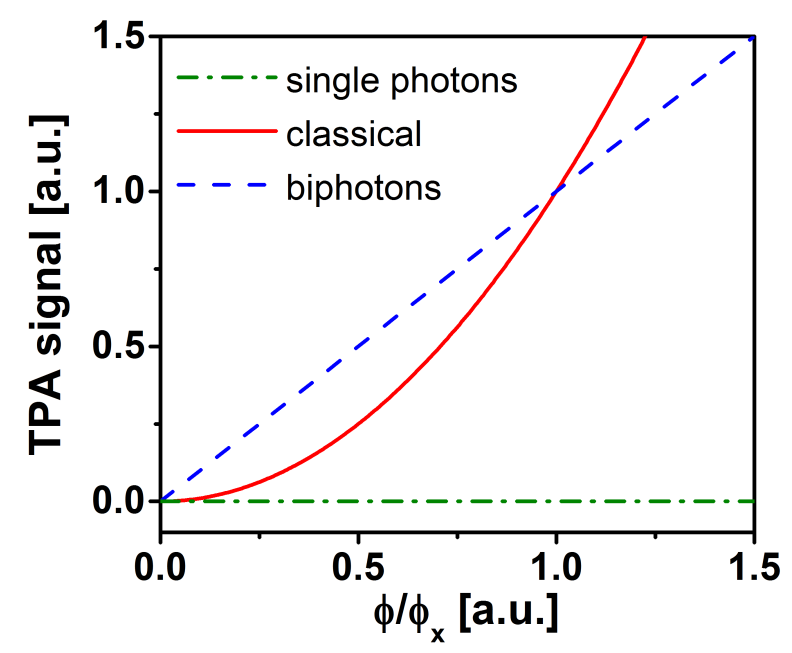

Figure B3. TPA rate $R_{T P A}$ as a function of photon flux $\Phi$ normalized to the critical photon flux $\Phi_{x}$ for single photons, biphotons and classical light.

the down-converted field, the linear part will dominate, while above $\Phi_{x}$ the quadratic, random term will dominate. The rate enhancement is [92]:

$$
\frac{R_{T P A, \text { correlated }}}{R_{T P A, \text { uncorrelated }}}=\frac{\Delta_{S P D C}}{\Delta_{T P A}} \cdot \frac{n^{2}-n}{n} \cdot \frac{P_{e}\left(\tau_{i}-\tau_{s}\right)}{g_{\text {pump }}^{(2)}(0)} .
$$

With $n$ being the photons per mode $\left(n=1\right.$ at $\left.\Phi_{x}\right), \Delta_{T P A}$ being the bandwidth of the twophoton process, $g_{\text {pump }}^{(2)}(0)$ the degree of second order coherence of the light field pumping the SPDC and $P_{e}$ being a normalized term describing a possible delay between signal and idler. Thus, at low excitation intensities (or small values of $n$ ) a drastic enhancement with nonclassical light is expected. Using a single photon source will result in no TPA signal.

Teich and Saleh proposed to exploit entangled photons for two-photon microscopy in 1997 [93] and later extended this idea towards virtual state spectroscopy [51]. Since a few years, several novel theoretical work has spawned in the context of entangled photon spectroscopy $[94,95,96]$. The recent tutorial by Schlawin is highly recommended for further reading [52].

\section{Acknowledgments}

The author would like to thank Ralf Menzel, Axel Heuer, Henning Kurzke and Jan Kiethe for helpful discussions in the framework of entangled two-photon absorption.

\section{Conflicts of Interest}

The author declares no conflict of interest. 
Towards heralded TPA and TPEF for quantum microscopy and spectroscopy

\section{References}

[1] Boyd R W 2008 Nonlinear Optics, Third Edition 3rd ed (Academic Press) ISBN 0123694701, 9780123694706

[2] Aspect A, Grangier P and Roger G 1982 Phys. Rev. Lett. 49(2) 91-94 URL http://link.aps.org/doi/10.1103/PhysRevLett.49.91

[3] Polycarpou C, Cassemiro K N, Venturi G, Zavatta A and Bellini M 2012 Phys. Rev. Lett. 109(5) 053602 URL http://link.aps.org/doi/10.1103/PhysRevLett.109.053602

[4] Manning A G, Khakimov R I, Dall R G and Truscott A G 2015 Nat Phys 11 539-542 ISSN 1745-2473 letter URL http://dx.doi.org/10.1038/nphys3343

[5] Dayan B, Parkins A S, Aoki T, Ostby E P, Vahala K J and Kimble H J 2008 Science 319 1062-1065 (Preprint http://www.sciencemag.org/content/319/5866/1062.full.pdf) URL http://www. sciencemag.org/content/319/5866/1062. abstract

[6] Chang D E, Vuletic V and Lukin M D 2014 Nat Photon 8 685-694 ISSN 1749-4885 review URL http://dx.doi.org/10.1038/nphoton.2014.192

[7] Göppert-Mayer M 1931 Annalen der Physik 401 273-294 ISSN 1521-3889

[8] Kaiser W and Garrett C G B 1961 Phys. Rev. Lett. 7(6) 229-231

[9] Denk W, Strickler J and Webb W 1990 Science 248 73-76

[10] Zipfel W R, Williams R M and Webb W W 2003 Nat Biotech 21 1369-1377 ISSN 1087-0156 URL http://dx.doi.org/10.1038/nbt899

[11] Dong J, Revilla-Sanchez R, Moss S and Haydon P G 2010 Neuropharmacology $\quad 59 \quad 268 \quad-\quad 275 \quad$ ISSN $0028-3908$ alzheimers Disease URL http://www.sciencedirect.com/science/article/pii/S0028390810001097

[12] Hopt A and Neher E 2001 Biophys J 80 2029-2036 ISSN 0006-3495 11259316[pmid] URL http://www.ncbi.nlm.nih.gov/pmc/articles/PMC1301392/

[13] Mollow B R 1968 Phys. Rev. 175(5) 1555-1563

[14] Boitier F, Godard A, Rosencher E and Fabre C 2009 Nat. Phys. 5(4) 267-270

[15] Jechow A, Seefeldt M, Kurzke H, Heuer A and Menzel R 2013 Nat. Photon. 7973

[16] Gea-Banacloche J 1989 Phys. Rev. Lett. 62(14) 1603-1606 URL http://link.aps.org/doi/10.1103/PhysRevLett.62.1603

[17] Javanainen $\mathrm{J}$ and Gould P L 1990 Phys. Rev. A 41(9) 5088-5091 URL http://link.aps.org/doi/10.1103/PhysRevA.41.5088

[18] Georgiades N P, Polzik E S, Edamatsu K, Kimble H J and Parkins A S 1995 Phys. Rev. Lett. 75(19) 3426-3429 URL http://link.aps.org/doi/10.1103/PhysRevLett.75.3426

[19] Lee D I, and Goodson III T 2006 The Journal of Physical Chemistry B 110 25582-25585 pMID: 17181189 (Preprint http://dx.doi.org/10.1021/jp066767g) URL http://dx.doi.org/10.1021/jp066767g

[20] Harpham M R, Süzer Ö, Ma C Q, Bäuerle P and Goodson III T 2009 Journal of the American Chemical Society 131 973-979 pMID: 19123819 (Preprint http://dx.doi.org/10.1021/ja803268s) URL http://dx.doi.org/10.1021/ja803268s

[21] Upton L, Harpham M, Süzer Ö, Richter M, Mukamel S and Goodson III T 2013 The Journal of Physical Chemistry Letters 4 2046-2052 pMID: 26283251 (Preprint http://dx.doi.org/10.1021/jz400851d) URL http://dx.doi.org/10.1021/jz400851d

[22] Villabona-Monsalve J P, Calderón-Losada O, Nuñez Portela M and Valencia A 2017 The Journal of Physical Chemistry A $\mathbf{1 2 1}$ 7869-7875

[23] Dayan B, Pe'er A, Friesem A A and Silberberg Y 2005 Phys. Rev. Lett. 94(4) 043602 URL http://link.aps.org/doi/10.1103/PhysRevLett.94.043602

[24] Jechow A, Lichtner M, Menzel R, Radziunas M, Skoczowsky D and Vladimirov A G 2009 Opt. Express 17 19599-19604

[25] U'Ren A B, Silberhorn C, Erdmann R, Banaszek K, Grice W P, Walmsley I A and Raymer M G 2005 Las. Phys. 15 146-161 
Towards heralded TPA and TPEF for quantum microscopy and spectroscopy

[26] Pan J W, Bouwmeester D, Weinfurter H and Zeilinger A 1998 Phys. Rev. Lett. 80(18) 3891-3894 URL http://link.aps.org/doi/10.1103/PhysRevLett.80.3891

[27] Hubel H, Hamel D R, Fedrizzi A, Ramelow S, Resch K J and Jennewein T 2010 Nature 466 601-603 ISSN 0028-0836 URL http://dx.doi.org/10.1038/nature09175

[28] Guerreiro T, Pomarico E, Sanguinetti B, Sangouard N, Pelc J S, Langrock C, Fejer M M, Zbinden H, Thew R T and Gisin N 2013 Nat Commun 4 article URL http://dx.doi.org/10.1038/ncomms3324

[29] Guerreiro T, Martin A, Sanguinetti B, Pelc J S, Langrock C, Fejer M M, Gisin N, Zbinden H, Sangouard N and Thew R T 2014 Phys. Rev. Lett. 113(17) 173601 URL http://link.aps.org/doi/10.1103/PhysRevLett.113.173601

[30] Spasibko K Y, Kopylov D A, Krutyanskiy V L, Murzina T V, Leuchs G and Chekhova M V 2017 Phys. Rev. Lett. 119(22) 223603 URL https://link.aps.org/doi/10.1103/PhysRevLett.119.223603

[31] Terenziani F, Katan C, Badaeva E, Tretiak S and Blanchard-Desce M 2008 Advanced Materials 20 4641-4678

[32] Wenseleers W, Stellacci F, Meyer-Friedrichsen T, Mangel T, Bauer C A, Pond S J, Marder S R and Perry J W 2002 The Journal of Physical Chemistry B 106 6853-6863

[33] Cohanoschi I, Yao S, Belfield K D and Hernández F E 2007 Effect of the concentration of organic dyes on their surface plasmon enhanced two-photon absorption cross section using activated au nanoparticles

[34] Lakowicz J R 2006 Plasmonics 1 5-33

[35] Streed E W, Norton B G, Jechow A, Weinhold T J and Kielpinski D 2011 Physical review letters 106010502

[36] Jechow A, Norton B, Haendel S, Blūms V, Streed E and Kielpinski D 2013 Physical review letters 110113605

[37] Jechow A, Streed E W, Norton B G, Petrasiunas M J and Kielpinski D 2011 Opt. Lett. 36 13711373 URL http://ol.osa.org/abstract.cfm?URI=ol-36-8-1371

[38] Streed E W, Jechow A, Norton B G and Kielpinski D 2012 Nature communications 3933

[39] Shu G, Kurz N, Dietrich M and Blinov B 2010 Physical Review A 81042321

[40] Maiwald R, Golla A, Fischer M, Bader M, Heugel S, Chalopin B, Sondermann M and Leuchs G 2012 Phys. Rev. A 86(4) 043431 URL http://link.aps.org/doi/10.1103/PhysRevA.86.043431

[41] Ghadimi M, Blūms V, Norton B G, Fisher P M, Connell S C, Amini J M, Volin C, Hayden H, Pai C S, Kielpinski D et al. 2017 npj Quantum Information 34

[42] Celebrano M, Kukura P, Renn A and Sandoghdar V 2011 Nat. Phot. 5 95-98

[43] Jechow A, Heuer A and Menzel R 2008 Opt. Express 16 13439-13449

[44] Sangouard N, Sanguinetti B, Curtz N, Gisin N, Thew R and Zbinden H 2011 Phys. Rev. Lett. 106(12) 120403 URL http://link.aps.org/doi/10.1103/PhysRevLett.106.120403

[45] Gregor M, Kuhlicke A and Benson O 2009 Optics express 17 24234-24243

[46] Alda I, Berthelot J, Rica R A and Quidant R 2016 Applied Physics Letters 109163105

[47] Streed E W 2012 arXiv preprint arXiv:1211.3168

[48] Norton B, Streed E, Petrasiunas M, Jechow A and Kielpinski D 2011 New Journal of Physics 13 113022

[49] VanDevender A P, Colombe Y, Amini J, Leibfried D and Wineland D J 2010 Phys. Rev. Lett. 105 023001

[50] Stefanov A 2017 Quantum Science and Technology 2025004

[51] Saleh B E, Jost B M, Fei H B and Teich M C 1998 Physical review letters 803483

[52] Schlawin F 2017 Journal of Physics B: Atomic, Molecular and Optical Physics 50203001

[53] Natarajan C M, Tanner M G and Hadfield R H 2012 Superconductor science and technology 25 063001

[54] Hanbury Brown R and Twiss R Q 1956 Nature 177 27-29

[55] Burnham D C and Weinberg D L 1970 Phys. Rev. Lett. 25(2) 84-87 URL 
Towards heralded TPA and TPEF for quantum microscopy and spectroscopy

http://link.aps.org/doi/10.1103/PhysRevLett.25.84

[56] Jost B, Sergienko A, Abouraddy A, Saleh B and Teich M 1998 Opt. Express 3 81-88 URL http://www .opticsexpress .org/abstract. cfm?URI=oe-3-2-81

[57] Harris S E, Oshman M K and Byer R L 1967 Phys. Rev. Lett. 18(18) 732-734 URL http://link.aps.org/doi/10.1103/PhysRevLett.18.732

[58] Malygin A A, Perin A N and Sergienko A V 1981 Sov. J. Quantum Electron. 11939

[59] Kwiat P G, Mattle K, Weinfurter H, Zeilinger A, Sergienko A V and Shih Y 1995 Phys. Rev. Lett. 75(24) 4337-4341 URL http://link.aps.org/doi/10.1103/PhysRevLett.75.4337

[60] Bouwmeester D, Pan J W, Mattle K, Eibl M, Weinfurter H and Zeilinger A 1997 Nature 390 575-579 ISSN 0028-0836 URL http://dx.doi.org/10.1038/37539

[61] Salter C L, Stevenson R M, Farrer I, Nicoll C A, Ritchie D A and Shields A J 2010 Nature 465 594-597 ISSN 0028-0836 URL http://dx.doi.org/10.1038/nature09078

[62] Wang L J, Hong C K and Friberg S R 2001 Journal of Optics B: Quantum and Semiclassical Optics 3346 URL http://stacks.iop.org/1464-4266/3/i=5/a=311

[63] Vallés A, Hendrych M, Svozilík J, Machulka R, Abolghasem P, Kang D, Bijlani B J, Helmy A S and Torres J P 2013 Opt. Express 21 10841-10849 URL http://www .opticsexpress.org/abstract. cfm?URI=oe-21-9-10841

[64] Boitier F, Orieux A, Autebert C, Lemaître A, Galopin E, Manquest C, Sirtori C, Favero I, Leo G and Ducci S 2014 Phys. Rev. Lett. 112(18) 183901 URL http://link.aps.org/doi/10.1103/PhysRevLett.112.183901

[65] Autebert C, Maltese G, Halioua Y, Boitier F, Lemaître A, Amanti M, Sirtori C and Ducci S 2016 Technologies 424

[66] Lounis B and Orrit M 2005 Reports on Progress in Physics 68 1129 URL http://stacks . iop.org/0034-4885/68/i=5/a=R04

[67] Mosley P J, Lundeen J S, Smith B J, Wasylczyk P, U'Ren A B, Silberhorn C and Walmsley I A 2008 Phys. Rev. Lett. 100(13) 133601 URL http://link.aps.org/doi/10.1103/PhysRevLett.100.133601

[68] Levine Z H, Fan J, Chen J, Ling A and Migdall A 2010 Opt. Express 18 3708-3718 URL http://www.opticsexpress.org/abstract.cfm?URI=oe-18-4-3708

[69] Wrigge G, Gerhardt I, Hwang J, Zumofen G and Sandoghdar V 2008 Nat Phys 4 60-66 ISSN 1745-2473 URL http://dx.doi.org/10.1038/nphys812

[70] Lee K G, Chen X W, Eghlidi H, Kukura P, Lettow R, Renn A, Sandoghdar V and Gotzinger S 2011 Nat Photon 5 166-169 ISSN 1749-4885 URL http://dx.doi.org/10.1038/nphoton.2010.312

[71] Keller M, Lange B, Hayasaka K, Lange W and Walther H 2004 Nature 431 1075-1078 ISSN 0028-0836 URL http://dx.doi.org/10.1038/nature02961

[72] McKeever J, Boca A, Boozer A D, Miller R, Buck J R, Kuzmich A and Kimble H J 2004 Science 303 1992-1994 (Preprint http://www.sciencemag.org/content/303/5666/1992.full.pdf) URL http://www.sciencemag.org/content/303/5666/1992.abstract

[73] Michler P, Imamoglu A, Mason M D, Carson P J, Strouse G F and Buratto S K 2000 Nature 406 968-970 ISSN 0028-0836 URL http://dx.doi.org/10.1038/35023100

[74] Kuhlmann A V, Prechtel J H, Houel J, Ludwig A, Reuter D, Wieck A D and Warburton R J 2015 Nat Commun 6

[75] Schnauber P, Thoma A, Heine C V, Schlehahn A, Gantz L, Gschrey M, Schmidt R, Hopfmann C, Wohlfeil B, Schulze J H et al. 2015 Technologies 41

[76] Zhang X, Jiang R, Bell B A, Choi D Y, Chae C J and Xiong C 2016 Technologies 425

[77] Boretti A and Rosa L 2016 Technologies 416

[78] Sipahigil A, Goldman M L, Togan E, Chu Y, Markham M, Twitchen D J, Zibrov A S, Kubanek A and Lukin M D 2012 Phys. Rev. Lett. 108(14) 143601 URL http://link.aps.org/doi/10.1103/PhysRevLett.108.143601

[79] Schietinger S, Barth M, Aichele $\mathrm{T}$ and Benson $\mathrm{O} 2009$ Nano Letters 9 16941698 pMID: 19301860 (Preprint http://dx.doi.org/10.1021/n1900384c) URL 
Towards heralded TPA and TPEF for quantum microscopy and spectroscopy

http://dx.doi.org/10.1021/n1900384c

[80] Albota M, Beljonne D, Brédas J L, Ehrlich J E, Fu J Y, Heikal A A, Hess S E, Kogej T, Levin M D, Marder S R, McCord-Maughon D, Perry J W, Röckel H, Rumi M, Subramaniam G, Webb W W, Wu X L and Xu C 1998 Science 281 1653-1656 (Preprint http://www. sciencemag.org/content/281/5383/1653.full.pdf) URL http://www. sciencemag. org/content/281/5383/1653.abstract

[81] Kano $\mathrm{H}$ and Kawata S 1996 Opt. Lett. 21 1848-1850 URL http://ol .osa.org/abstract. cfm?URI=ol-21-22-1848

[82] Sivapalan S T, Vella J H, Yang T K, Dalton M J, Swiger R N, Haley J E, Cooper T M, Urbas A M, Tan L S and Murphy C J 2012 Langmuir 28 9147-9154 pMID: 22500968 (Preprint http://dx.doi.org/10.1021/la300762k) URL http://dx.doi.org/10.1021/la300762k

[83] Kühn S, Håkanson U, Rogobete L and Sandoghdar V 2006 Phys. Rev. Lett. 97(1) 017402 URL http://link.aps.org/doi/10.1103/PhysRevLett.97.017402

[84] Teich M C and Wolga G J 1966 Phys. Rev. Lett. 16(14) 625-628

[85] Lambropoulos P, Kikuchi C and Osborn R K 1966 Phys. Rev. 144(4) 1081-1086

[86] Shen Y R 1967 Phys. Rev. 155(3) 921-931

[87] Agarwal G S 1970 Phys. Rev. A 1(5) 1445-1459

[88] Jechow A, Raab V and Menzel R 2008 Applied optics 47 1447-1450

[89] Kiethe J, Heuer A and Jechow A 2017 Laser Physics Letters 14086201

[90] Kurzke H, Kiethe J, Heuer A and Jechow A 2017 Laser Physics Letters 14055402

[91] Aßmann M, Veit F, Bayer M, van der Poel M and Hvam J M 2009 Science 325 297-300

[92] Dayan B 2007 Physical Review A 76043813

[93] Teich M C and Saleh B E 1997 Cesk. Cas. Fyz 47 3-8

[94] Schlawin F, Dorfman K E, Fingerhut B P and Mukamel S 2012 Physical Review A 86023851

[95] Schlawin F, Dorfman K E, Fingerhut B P and Mukamel S 2013 Nature communications 41782

[96] Dorfman K E, Schlawin F and Mukamel S 2016 Reviews of Modern Physics 88045008 